\title{
VIRTUAL SOUND
}

\section{Dear Reader,}

The automotive industry is continually being subjected to dynamic developments, which themselves result in the development of new technologies. An issue that is currently acute is the need to reduce fuel consumption and $\mathrm{CO}_{2}$, but without neglecting driving enjoyment. Downspeeding and downsizing - or rather: "rightsizing" - are the latest measures being applied to improve the efficiency of internal combustion engines while at the same time improving vehicle performance. However, these technologies have a significant influence on the NVH characteristics of vehicles and therefore pose new challenges on vehicle acoustics. Active Noise Cancellation (ANC) and Active Sound Design (ASD) are two examples of such technologies that might offer a solution, and when supported by a special sound design they can also secure the key component of the core attributes of a brand, such as sportiness.

The latest issue of ATZ examines the subject of NVH in detail. For example, the cover story describes how Eberspächer uses electro-magnetic actuators in its ActiveSilence system to actively influence the sound field by means of a special software package. As the overall ANC system has meanwhile become much more robust, efficient and powerful, its use in diesel engines can now be extended to more demanding applications in spark-ignition engines. As a result, active silencers in the premium car market are no longer a pipe dream.

Lightweight design is also an important means of cutting fuel consumption, but it can also lead to unwanted vibration as masses are reduced. One solution is to use intelligent components. In the EAPSIG research project, the Institute of Automotive Engineering (ika) at RWTH Aachen University, together with its project partner Vemac, is working on implementing Active Vibration Control (AVC) on the basis of electro-active polymers. Combining a component in this case a vehicle bonnet was used as an example - with the adaptive actuator can reduce both unwanted vibration and vehicle mass. Once testing on the component level is complete, the plan is to test the AVC system on the complete vehicle.

These are just two of the exciting new developments examined in the usual depth in your latest issue of ATZ. Even more information will be available at our ATZlive Automotive Acoustics Conference on the subject of Acoustics/NVH on 26 and 27 June 2013 in Zurich. It is good to know that, in spite of all the necessary reduction in fuel consumption, improvements in efficiency and the optimisation of acoustics, the car will still remain what it always has been: an object of high emotionality - even though its sound might be virtual.

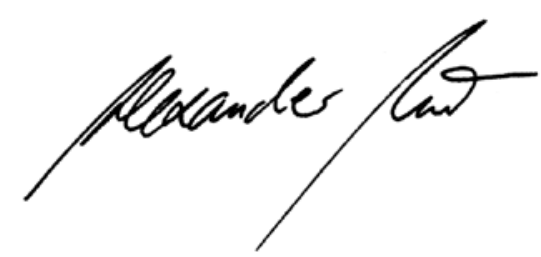

DR. ALEXANDER HEINTZEL, Editor in Chief Wiesbaden, 17 May 2013

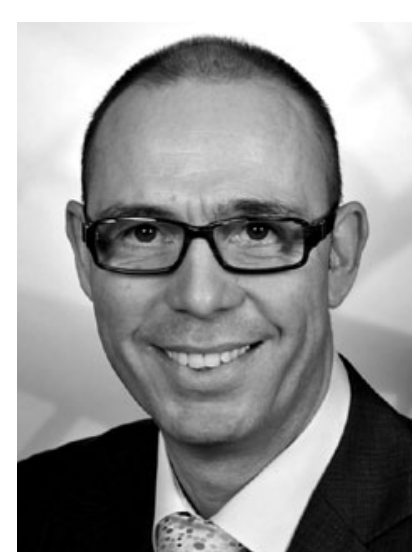

